\title{
Le constructivisme radical et Socrate : aspects pédagogiques et idéologiques
}

\section{Alberto Martínez Delgado}

\section{OpenEdition}

\section{Journals}

Édition électronique

URL : http://journals.openedition.org/trema/2863

DOI : $10.4000 /$ trema.2863

ISSN : 2107-0997

Éditeur

Faculté d'Éducation de l'université de Montpellier

Édition imprimée

Date de publication : 1 décembre 2012

Pagination : 56-85

ISBN : 1167-315X

ISSN : $1167-315 X$

Référence électronique

Alberto Martínez Delgado, «Le constructivisme radical et Socrate : aspects pédagogiques et

idéologiques », Tréma [En ligne], 38 | 2012, mis en ligne le 01 décembre 2014, consulté le 30 avril 2019. URL : http://journals.openedition.org/trema/2863 ; DOI : 10.4000/trema.2863

Ce document a été généré automatiquement le 30 avril 2019.

Trema 


\section{Le constructivisme radical et Socrate : aspects pédagogiques et idéologiques ${ }^{1}$}

Alberto Martínez Delgado

I. Le constructivisme radical et son mépris pour Socrate 
1 Socrate a bénéficié d'une bienveillance générale de la part de la tradition philosophique et pédagogique, notamment en raison de l'utilisation du dialogue pour faire émerger la connaissance. Par ailleurs, nous pouvons remarquer des coïncidences importantes entre le constructivisme radical et les positions de Socrate, tant en ce qui concerne les principes épistémologiques généraux que les précisions et applications éducatives.

2 Dans ce contexte, le mépris du constructivisme radical pour la conception

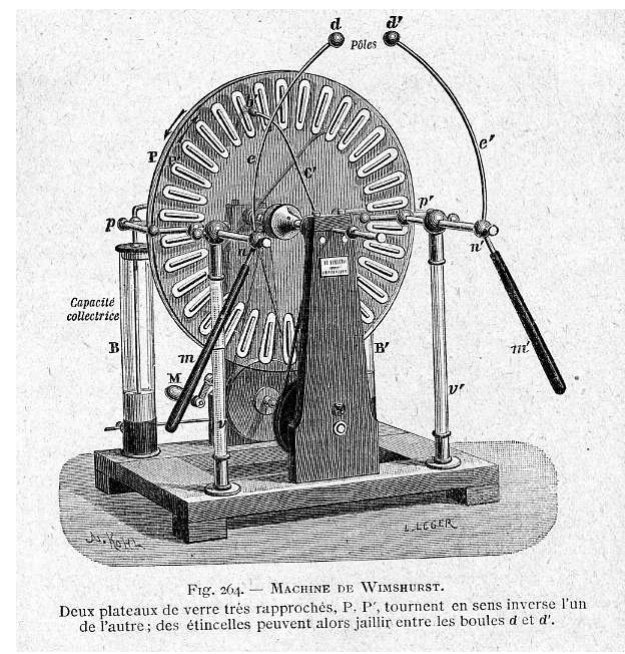
socratique de la connaissance et de ses méthodes pédagogiques est surprenant. Von Glasersfeld ${ }^{2}$ situe l'origine des problèmes traditionnels à propos de la théorie de la connaissance chez Socrate et mentionne les sophistes Protagoras et Gorgias comme des initiateurs de l'alternative constructiviste.

3 La principale référence de von Glasersfeld à la théorie socratique que nous avons trouvée montre ce mépris pour la maïeutique socratique, qu'il assimile à un constructivisme trivial $^{3}$, basé seulement sur le premier des deux principes qui définissent le constructivisme de von Glasersfeld (1991, p. 31) :

4 En pratique, le premier principe formulé par von Glasersfeld est admis par de nombreux auteurs comme le seul véritable principe constructiviste, bien que, comme von Glasersfeld le reconnait, il n'apporte pratiquement rien de nouveau ${ }^{4}$. À notre avis, le second principe mentionné par von Glasersfeld ne relève pas non plus d'une nouveauté importante et n'ajoute aucune avancée radicale conséquente au premier, puisqu'en qualifiant la réalité d'ontologique apparaît une équivoque, si von Glasersfeld se réfère à une réalité au sens métaphysique du mot - ce qui serait refusé aussi par la plupart des tendances réalistes et matérialistes - ou à tout type de réalité, extérieure au sujet, au sens ordinaire ou au sens scientifique du mot, ce qui mènerait, malgré quelques hésitations et protestations de von Glasersfeld, au solipsisme.

5 La radicalité constructiviste apparaît, de notre point de vue, dans d'autres énoncés où l'on fait du sujet le seul acteur de la connaissance et de la réalité une création du sujet.

6 Nola (1998, p. 36) montre quant à lui son étonnement devant l'oubli constructiviste des approches épistémologiques de Socrate-Platon :

\section{Les principes socratiques et constructivistes: coïncidences et différences épistémologiques}

7 La théorie épistémologique élaborée par Socrate a en commun avec le constructivisme radical le principe fondamental selon lequel la connaissance ne s'acquiert pas à partir d'une réalité extérieure à l'individu, mais qu'elle est un produit interne de l'individu même. Dans le constructivisme, le sujet invente ses propres connaissances, alors que d'après la théorie socratique, les connaissances sont encapsulées de façon codifiée, dans 
le sujet, dans sa partie spirituelle, dans l'âme. Dans les deux cas, il existe un processus d'invention ou d'explicitation de la connaissance de la part du sujet qui, dans le constructivisme radical, renvoie à la propre initiative de l'individu en accord avec des schémas personnels d'action et de structuration (associés à une incitation méthodologique constructiviste), et dans la théorie socratique, constitue un processus de remémoration se faisant jour à l'intérieur du sujet, au travers d'un dialogue mené à bon escient. La remémoration socratique peut être vue comme un processus de construction ou de reconstruction de la connaissance, là où celle-ci est accouchée (d'où le terme de maïutique) grâce à la composition des idées préalables - si appréciées dans la pédagogie constructiviste - du sujet. D'autre part, la construction envisagée par le constructivisme radical peut être vue comme le résultat de l'action d'éléments personnels antérieurs qui se manifestent, se remémorent, comme des cadres «conceptuels et perceptuels » du sujet.

8 L'immortalité de l'âme socratique-platonicienne peut suggérer une certaine immuabilité de l'âme qui puisse aussi se transmettre à la connaissance qu'on lui attribue, de sorte que cette connaissance soit une connaissance absolue et éternelle, en contraste avec l'incertitude des constructions individuelles. Cette interprétation absolue de la connaissance, en accord avec le caractère divin de l'âme impliquerait une distanciation considérable entre les principes épistémologiques socratique-platoniciens et ceux du constructivisme radical contemporain. Toutefois, cette interprétation absolutisante de l'âme platonicienne, et des idées ou formes qui en font partie, n'est pas évidente ni constante dans les textes platoniciens, en particulier dans le Ménon.

Cinq points, à notre avis, remettent en question cette interprétation. Tout d'abord, la difficulté d'utiliser des arguments ou des images absolus pour traiter des faits relatifs comme le sont la plupart des questions abordées dans les dialogues de Platon (peut-être cette difficulté se présente, en général, dans toutes les tentatives d'argumenter à partir d'une idée de l'absolu). Deuxièmement, il convient de noter les variations de la pensée de Socrate-Platon sur la nature de l'âme. Les troisième et quatrième points sont liés aux dimensions respectivement spatiales et temporelles de l'âme, lesquelles sont abordées dans le Ménon.

10 Les différentes âmes, ancrées dans des corps différents, ont des caractéristiques différentes entre elles et des compositions aussi différentes selon les parties du corps auxquelles elles sont associées: la tête, le cœur et le ventre. Cette structure tripartite du corps et de l'âme est aussi proposée par Platon pour son modèle de société, divisée en trois classes sociales (les philosophes, les guerriers et les artisans, en accord avec la prédominance de pulsions provenant de la tête, du cœur ou du ventre). Cette structure sociale, du point de vue d'une herméneutique du soupçon, est révélatrice de l'arrière-plan idéologique de classe de la théorie platonicienne.

11 Le corps dans lequel chaque âme est déposée agit comme une prison pour celle-ci et a un impact sur elle, embrumant son entendement et sa relation avec le monde des idées. Selon ces conditions qui peuvent être considérées comme spatiales, les différentes âmes auraient différents niveaux de connaissances et différentes possibilités d'accès ou de remémoration des connaissances.

Les changements temporels des âmes sont liés non seulement aux évolutions au cours de la vie des individus mais aussi à leurs incarnations successives, dont chacune peut modifier le degré d'interpénétration de chaque âme avec le monde des idées, des formes 
ou des essences, bien que l'on puisse soutenir que les moments décisifs de ce contact se produisent après les morts successives des corps. Russell $(1979$, p. 153) soutient cette interprétation de la modification existentielle des âmes et donc de la connaissance : « La même conclusion est obtenue dans le Ménon et le Phédon, la connaissance est recueillie dans l'âme à partir d'une existence antérieure ». Socrate, dans le Ménon (Platon, 1993, p. 153, 81c-d), indique comme source de la connaissance la contemplation purement spirituelle de l'âme, séparée du corps : Enfin, les implications épistémologiques de l'immortalité de l'âme socratique-platonicien ne sont confinées qu'à une partie de la connaissance, laquelle peut être considérée, jusqu'à un certain point, comme étrangère à la connaissance scientifique de la nature physique et humaine (connaissance empirique) et se réduit au domaine des connaissances qui sont évidentes ou que l'on peut obtenir par déduction à partir de ces idées évidentes. Ce genre de connaissance, devenant mystique si on l'absolutise, favorise une vision idéaliste avec des ambiguïtés et des oscillations qui entravent la formation d'une théorie cohérente de la nature et de la connaissance humaine.

Cette autolimitation de l'épistémologie platonicienne, les avatars des âmes, soumises a des purifications lors des réincarnations successives, l'exhortation de Socrate à prendre soin de son âme (Alcibiade) et les différences entre les descriptions des mythes platoniciens, affaiblissent la vision de la doctrine platonicienne comme une théorie cohérente et rigoureuse qui puisse nettement s'opposer à d'autres théorisations idéalistes, telles que le constructivisme radical.

L'ambiguïé de l'importance cognitive de la vie terrestre des âmes et de la connaissance empirique dans l'œuvre platonicienne est soulevée par Canto-Sperber (1993, p. 80) :

Selon nous, le réalisme idéaliste de l'épistémologie de Platon n'est pas un obstacle insurmontable à son rapprochement avec l'épistémologie constructiviste radicale, en dépit de la dérive solipsiste inhérente à cette dernière. De même, le constructivisme radical peut être intégré dans une perspective platonicienne.

Le constructivisme, radical ou non, même s'il ne l'affirme pas explicitement, présente les constructions-connaissances individuelles comme des approximations d'une connaissanceréalité préétablie qui, si l'on ne la met pas en connexion avec une réalité matérielle, est vouée à rejoindre une vue similaire à celle de l'idéalisme platonicien - et en ceci il coïncide avec d'autres paradigmes épistémologiques tels que le réalisme. La relation entre les constructions individuelles et la connaissance préétablie - résultant de l'interaction des individus et des groupes sociaux avec la nature et entre eux-mêmes - est asymétrique, avec une prédominance du savoir préétabli auquel, en général, doivent s'accommoder les tâtonnements cognitifs des individus. Dans une orientation constructiviste radicale, en suivant des mécanismes indirectes de modélisation - en dépit de sa conception de la connaissance comme non transmissible - on essaie de faire émerger des connaissances préexistantes, d'une façon semblable à celle suivie par l'âme platonicienne, emprisonnée et limitée par un corps matériel, pour s'approcher de la connaissance de l'idée pure.

19 La différence principale entre ces deux modèles épistémologiques est due au réalisme idéaliste de Socrate (tel qu'il est présenté par Platon). L'idée de l'existence d'une connaissance stable et permanente de l'âme, que le processus d'anamnèse parvient à mettre en lumière dans toute sa plénitude, peut conduire à l'idée, rejetée par le constructivisme radical, de l'existence d'une réalité extérieure au sujet (ou, à la rigueur, 
au corps du sujet). Cependant, du point de vue de l'idéalisme socratique, l'âme ne peut sans doute pas être envisagée comme quelque chose d'extérieur au sujet, mais comme sa partie proprement humaine, rationnelle, de sorte que sa potentialité cognitive n'est pas étrangère au sujet, mais en est le noyau. En ce sens, nous retrouverions une convergence entre la théorie socratique et le constructivisme radical. Cette convergence est envisageable également si l'on considère que les présupposés de la rationalité et de la conscience humaine du constructivisme radical sont des notions aussi éthérées et insaisissables que celles de l'idée socratique de l'âme.

Si pour le constructivisme radical, la connaissance est inventée par le sujet au travers de sa propre rationalité et constitue une activité consciente, en marge de la réalité extérieure, le saut unificateur de la rationalité et de la conscience à l'âme socratique semble fondamentalement terminologique.

21 Les ambiguïtés des idées platoniciennes sont à mettre en corrélation avec les ambiguïtés du constructivisme radical, surtout avec ses oscillations entre le solipsisme et des formes subreptices de réalisme. Le constructivisme radical, malgré son refus de l'importance épistémologique de la réalité extérieure à l'individu et son penchant vers le solipsisme, glisse aussi vers la reconnaissance de la réalité objective (Martínez Delgado, 2002), soit sous la forme de construction sociale soit sous la forme d'une élimination des tentatives et des idées erronées des individus 5 .

La proximité entre les principes socratiques et constructivistes radicaux se manifeste aussi dans ses difficultés à répondre à des objections visant ces deux conceptions. Ainsi, tant dans la théorie socratique que dans le constructivisme radical, il existe une difficulté délicate à résoudre, le paradoxe de Ménon (Platon, 1993, p. 152) :

La solution de Socrate à ce paradoxe dit «de l'acquisition » et proposé par Ménon est doublement évasive : d'une part, Socrate fait appel, de façon quelque peu confuse, à la " vérité » et à la beauté des " choses divines ", au langage des " prêtres et des prêtresses " qui « déclarent en effet que l'âme de l'homme est immortelle, et que tantôt elle arrive à un terme - c'est justement ce qu'on appelle " mourir » -, tantôt elle nait à nouveau, mais qu'elle n'est jamais détruite» (p. 153); d'autre part, Socrate fait allusion aux conséquences pragmatiques négatives de « cet argument heuristique » : « il nous rendrait paresseux [...] tandis que l'argument que j'ai rapporté exhorte au travail et rend ardent à chercher » (p. 154). La réponse de Socrate implique de faire taire l'adversaire, en utilisant l'irrationalité religieuse hégémonique et, en fin de compte, la peur d'affronter les structures politico-religieuses et les valeurs socio-productives dominantes, une peur qui n'a rien d'imaginaire comme l'a démontré un peu plus tard, la condamnation à mort de Socrate.

24 La réponse du constructivisme radical au paradoxe de Ménon ne semble guère avoir eu un meilleur sort que celle apportée par Socrate.

Les frontières entre l'idéalisme objectif socratico-platonicien et l'idéalisme subjectif du constructivisme radical sont en grande partie diluées non seulement dans la mise en œuvre éducative des principes généraux mais aussi dans les nuances apportées aux dits principes. 


\section{Constructivisme et maïeutique en éducation : le Ménon, une fraude intellectuelle?}

L'extrait du Ménon où Socrate prétend résoudre le problème géométrique consistant à déterminer un carré ayant une surface du double de la surface d'un autre carré donné (dont le côté est de deux unités) est une puissante illustration de la théorie socratique de la connaissance. La solution apportée par Socrate est considérée par Matthews (1995, p. 94) comme « peut-être l'un des épisodes d'enseignement les plus connus de tous les temps [...] [et] une merveilleuse démonstration de la Méthode Socratique, une démonstration qui a été citée, tout au long des siècles suivants, comme un modèle exemplaire de bon enseignement ».

Dans le Ménon, après que l'esclave et Socrate expriment leur accord sur la solution au problème, Socrate pose la question « il y a-t-il une opinion que ce garçon ait donnée en réponse, qui ne vînt pas de lui ", Ménon répond "non, au contraire, tout venait de luimême » (Platon, 1993, p. 168). Ce dialogue possède clairement une teneur constructiviste ${ }^{6}$.

Le constructivisme radical, en éducation, coïncide avec la méthode socratique en ce qu'il accorde une importance primordiale aux idées préexistantes dans l'individu (dans le Ménon, la connaissance de la langue et le concept de surface d'un carré, fondamentalement), idées individuelles préexistantes qui doivent être modifiées et s'approcher ou coïncider avec les idées et connaissances préétablies (ou avec la réalité idéale du platonisme). En principe cette attention prioritaire accordée aux idées préexistantes est cohérente avec les prémisses socratico-constructivistes générales, mais elle implique en même temps l'apparition de critères d'objectivité réaliste en ce qui concerne l'action de Socrate - ou du professeur - pour faciliter la construction ou la remémoration en utilisant une connaissance objective des processus mentaux de l'esclave.

Après deux tentatives de l'esclave pour trouver le carré dont la surface serait le double de celle d'un autre carré de deux pieds de côté -, ayant donné successivement les réponses spontanées de quatre et trois pieds, l'esclave - comme la plupart des gens qui ne connaissent pas ce problème - se sent déconcerté, il avoue ne pas connaître la solution et essaie de se sortir de cette situation inconfortable («Mais par Zeus, Socrate, je ne le sais pas»). Finalement, Socrate rentre dans la phase finale du processus de remémoration, (Platon, 1993, p. 165-168), dans laquelle l'illustration graphique (résumée ici dans le dessin joint au dialogue verbal) semble jouer un rôle primordial : 


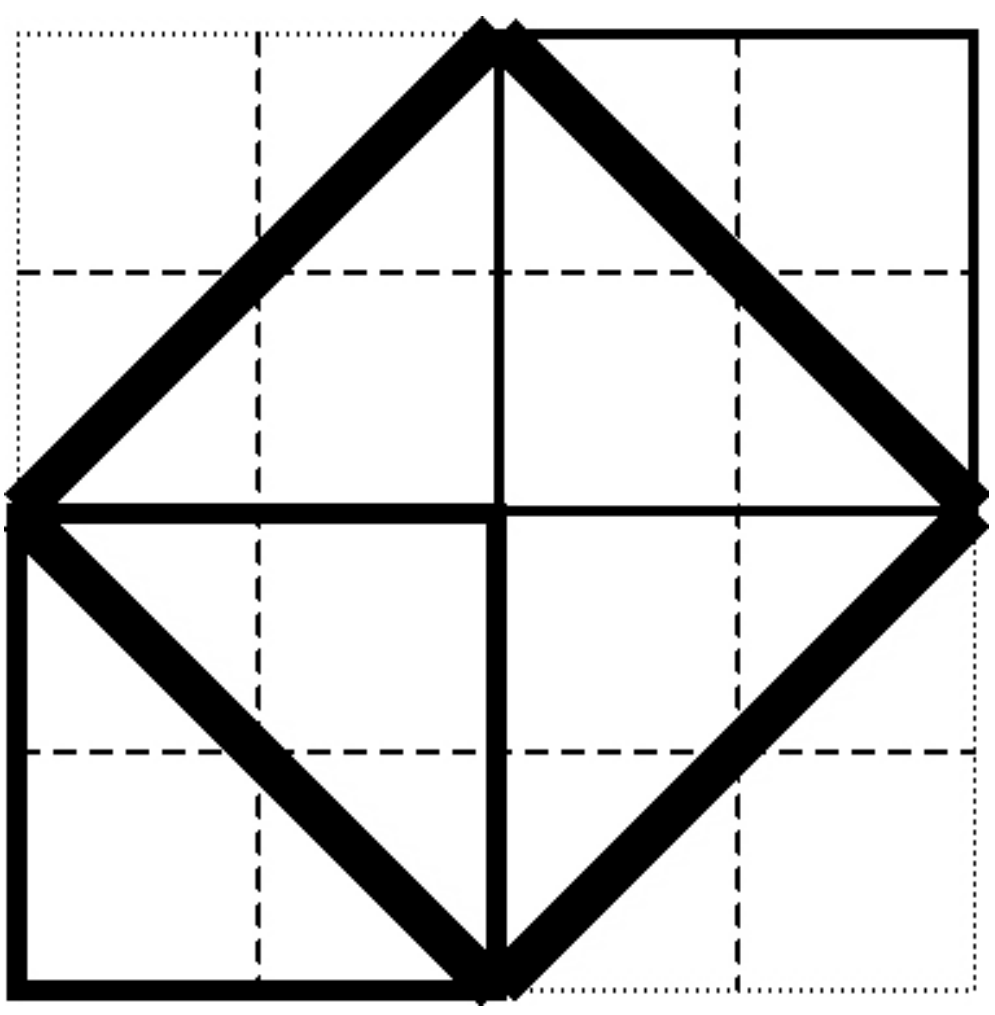

- Socrate: ... Dis-moi donc, mon garçon, n'avons-nous pas là un espace de quatre pieds carrés ? Comprends-tu?

[- Réponse : Oui, je comprends].

- Socrate : Pourrions-nous lui ajouter cet autre espace, qui lui est égal ?

[- Réponse : Oui].

- Socrate : Et aussi ce troisième espace qui est égal à chacun des deux autres ?

[- Réponse : Oui].

- Socrate : En ce cas, nous pourrions combler cet espace-ci dans le coin ?

[- Réponse : Oui, tout à fait].

- Socrate : Les quatre espaces que voici, ne seraient-ils pas égaux?

[- Réponse : Oui].

- Socrate: Que se passe-t-il alors ? Ce tout qu'ils forment, de combien de fois est-il plus grand que cet espace-ci?

[- Réponse : Quatre fois plus grand].

- Socrate: Mais il nous fallait obtenir un espace deux fois plus grand, ne t'en souviens-tu pas?

[- Réponse : Oui, tout à fait].

- Socrate: Or n'a-t-on pas ici une ligne qui va d'un coin à un autre coin et coupe en deux chacun de ces espaces?

[- Réponse : Oui].

- Socrate: Et n'avons nous pas là quatre lignes, qui sont égales, et qui enferment cet espace-ci?

[- Réponse : Oui, nous l'avons]. 
- Socrate : Eh bien, examine la question : quelle est la grandeur de cet espace?

[- Réponse : Je ne comprends pas].

- Socrate: Prenons ces quatre espaces qui sont là, chaque ligne en divise-t-elle pas chacun d'eux, à l'intérieur, par la moitié ? N'est-ce pas le cas ?

[- Réponse : Oui].

- Socrate : Or combien de surfaces de cette dimension se trouvent dans ce carré-ci ?

[- Réponse : Quatre].

- Socrate : Et combien dans ce premier espace?

[- Réponse : Deux].

- Socrate : Mais combien de fois deux font quatre?

[- Réponse : Deux fois].

- Socrate : Donc ce carré, combien a-t-il de pieds?

[- Réponse : Huit pieds carrés].

- Socrate : Sur quelle ligne est-il construit?

[- Réponse : Sur celle-ci].

- Socrate : Sur la ligne qu'on trace d'un coin à l'autre d'un carré de quatre pieds ?

[- Réponse : Oui].

- Socrate: C'est justement la ligne à laquelle les savants donnent le nom de « diagonale». En sorte que, si cette ligne s'appelle bien « diagonale », ce serait à partir de la diagonale que, d'après ce que tu dis, serviteur de Ménon, on obtiendrait l'espace double

[- Réponse : Oui, parfaitement, Socrate] (C'est nous qui soulignons).

Après avoir repris avec toute la fidélité possible, l'extrait décisif de l'argumentation sur la remémoration de la connaissance, une question fondamentale s'impose : Est-il vrai que la réponse au problème soit apportée par l'esclave à partir de ses propres connaissances que " tout venait de lui-même?", comme l'affirme Platon dans le Ménon (selon une citation antérieure).

31 L'affirmation de Socrate selon laquelle le jeune esclave a obtenu par lui-même la solution au problème proposé, à la lumière du texte du dialogue, des graphiques et légendes utilisées en complément, nous semble peu crédible, voire frauduleuse ${ }^{7}$. Le moment de la relation Socrate-esclave où Socrate montrant la figure trace la diagonale du carré initial et signale une opération similaire dans les autres trois répétitions du dit carré, moment que nous avons mis en relief dans le texte cité, montre clairement à notre avis que la solution est communiquée à l'esclave, qui n'a d'autre participation active que de vérifier qu'il s'agit effectivement de la solution.

Finalement, il est important de remarquer un autre aspect discutable aussi de l'énoncé et de la solution apportée par Socrate au problème qu'il a proposé. Après les premiers tâtonnements, Socrate formule la question qui définit le problème (Platon, 1993, p. 157) :

Bien que plus tard Socrate abandonne la question sur la « longueur", se concentrant sur la ligne qui sert de côté au carré recherché, cette question formulée initialement est celle à laquelle l'esclave essaie de répondre dans ses deux premières tentatives de solution (quatre pieds et trois pieds, respectivement) et c'est cette même question qui n'obtient pas de réponse dans l'ensemble de l'œuvre. 
Un problème d'intérêt multiple surgit ici : la réponse à la longueur du côté du carré $(2 \sqrt{2})$, est obtenue en appliquant le théorème de Pythagore (à partir du côté initial de deux unités considéré dans le Ménon), ce qui fait apparaitre des concepts nouveaux et des relations nouvelles du point de vue de l'enseignement, mais, d'un autre côté, l'application du théorème de Pythagore dans ce cas, conduit à l'apparition de nombres irrationnels (la mesure du côté du carré cherché ne peut être exprimée avec exactitude par une fraction ou raison entre deux nombres entiers). Ces nombres irrationnels ont infligé dans le monde mathématico-philosophique grec un démenti à une grande partie de ses idées sur les proportions du monde et des structures sociales, jusqu'au point, semble-t-il, d'avoir provoqué l'interdiction au sein de la secte pythagoricienne de rendre publique l'existence de tels nombres irrationnels (non exprimables par une fraction), et d'avoir condamné, selon quelques récits légendaires, à la peine de mort le membre de la secte Hippasus, pour avoir désobéi à une interdiction aussi obscurantiste.

La figure platonicienne de Socrate, bien que la formulation initiale du problème à résoudre invitât à rentrer dans la question des nombres irrationnels, se détourne de l'énoncé initial, changeant fondamentalement l'énoncé du problème, et laisse dans l'ombre une affaire si intéressante, qui contredit la théorie des proportions de l'idéalisme pythagoricien et platonicien. Cette contradiction est à la base du nom arreton, ineffable, donné par les Grecs à ce nouveau type de nombres, et peut expliquer aussi le silence dans le Ménon sur la réponse numérique au problème initialement posé. Cette opération de détournement subreptice de l'objectif à poursuivre est un indice de plus d'un processus intellectuel pour le moins douteux, avec de présumables connotations socio-idéologiquesreligieuses profondes, qui peut être aussi perçu dans le développement des théories constructivistes radicales.

Plusieurs objections à la théorie de la réminiscence socratique peuvent aussi être adressées au constructivisme radical, comme par exemple la difficulté d'expliquer le caractère cumulatif de la connaissance et ses progrès historiques.

\section{Le constructivisme de Kelly et de Piaget : les constructions réalistes}

7 Le constructivisme radical, avons-nous soutenu, déforme l'épistémologie de Socrate afin de prendre ses distances par rapport à celle-ci. Dans le cas de Kelly et Piaget, le constructivisme radical opère également une distorsion de leurs théories, mais cette fois afin de les intégrer - l'intégration de la théorie de Piaget contribuant au prestige du constructivisme radical.

Kelly fait partie, avec Piaget, des auteurs revendiqués par le constructivisme radical en tant qu'initiateurs de cette orientation épistémologique. Cependant, dans son principal ouvrage, de 1955, The Psychology of Personal Constructs, Kelly adopta clairement une épistémologie réaliste dans le traitement des constructions humaines, utilisant aussi le verbe to fit, revendiqué par von Glasersfeld, dans un sens réaliste (1955, p. 6, 8-9) :

Bien qu'à d'autres moments, Kelly $(1969$, p. 227) se soit déclaré comme non réaliste, il est très clair que le lien entre l'attention portée aux constructions mentales des individus et le déni de la pertinence épistémologique de la réalité extérieure n'est pas nécessaire.

Piaget, invoqué comme un pilier du constructivisme radical par les défenseurs de cette construction théorique fait, cependant, de nombreuses déclarations explicitement 
réalistes. En contraste évident avec l'intégration de l'expérience au sujet, Piaget en arrive à présenter le sujet comme une partie de cette réalité objective, se rapprochant ainsi d'une approche épistémologique matérialiste (1974/1985, p. 274) :

Des déclarations de principe de ce type, abondantes dans l'œuvre de Piaget, bien que parfois l'accent mis sur l'action du sujet semble oublier l'objet extérieur, n'empêchent pas von Glasersfeld (1996, p. 4-5) d'interpréter Piaget dans une perspective constructiviste :

Dans la citation précédente, von Glasersfeld semble se prononcer pour l'élimination de l'influence de la réalité extérieure, la réduction de la connaissance et de l'action du sujet à une insaisissable interaction entre des états successifs de l'individu selon un processus strictement interne.

Le rôle de l'environnement extérieur dans la conception évolutionniste de Piaget ne se réduit pas simplement à une action de sélection. Cette conception a même été considérée comme une forme de lamarckisme. Bien que Piaget se soit défendu d'être lamarckiste, les expériences qu'il a réalisées dans des lacs suisses et l'étude d'autres expériences l'ont conduit à retenir la possibilité que certains traits acquis par les individus d'une espèce sous la pression de certaines conditions environnementales (phénotype), puissent devenir héréditaires, devenant partie intégrante du patrimoine génétique de l'espèce (génotype), produisant ainsi un phénomène de phénocopie.

Piaget, opposé à la théorie dominante de l'évolution biologique, le néo-darwinisme orthodoxe - selon lequel l'évolution de l'espèce est indépendante de l'adaptation à l'environnement des individus de cette espèce -, soutient l'existence d'un certain impact des conditions environnementales sur le génotype (Piaget, 1974/1978, p. 30).

La distance entre les positions les plus établies de Piaget et l'interprétation faite par von Glasersfeld peut être perçue très clairement dans la citation suivante de Piaget (1974/1978, p. 76), où il rejette l'insistante position de von Glasersfeld sur l'idée que la réalité extérieure (acceptée avec réticence) agisse exclusivement en éliminant les tentatives du sujet :

Piaget établit un parallélisme et un prolongement entre les phénomènes biologiques et cognitifs ${ }^{8}$, en présentant ces derniers dans la perspective des processus d'assimilationaccommodation - il s'agit là de l'un des points discutables de sa théorie. Le constructivisme radical s'appuie aussi sur ce modèle biologique de l'évolution et transpose la nette séparation néo-darwinienne entre le patrimoine génétique et l'environnement, à une déconnexion totale entre le sujet et ses connaissances, d'une part et la réalité extérieure, d'autre part. On rejette ainsi l'influence de l'environnement extérieur, non seulement comme le néodarwinisme sur les espèces, mais aussi sur l'individu; il en résulte un déplacement de considérations biologiques phylogénétiques néodarwinistes sur le terrain du développement et de l'apprentissage des individus, forçant dogmatiquement un parallélisme entre des instances distinctes qui même si elles peuvent présenter certaines analogies ne peuvent être réduites de façon simpliste à une seule catégorie de phénomènes. La filiation piagétienne n'empêche pas, cependant, que certaines idées de Piaget comme celles de " phénocopie cognitive » et de "connaissance exogène » (Piaget, 1974/1978, p. 155) se voient reléguées par le constructivisme radical.

La déformation par von Glasersfeld des positions épistémologiques de Piaget concerne aussi le rôle socio-académique joué par Piaget, le présentant sous l'angle d'une certaine victimisation, comme un auteur marginalisé par les grands courants épistémologiques du XXe siècle, alors que la réalité est bien différente: Piaget a été l'un des chercheurs sur la 
connaissance et l'éducation ayant exercé le plus d'influence sur le monde académique et sur les administrations éducatives?.

\section{Constructivisme, enseignement et recherche scientifique}

\section{1 Attention au sujet dans l'enseignement : la pédagogie active} rupture avec le dogmatisme scolastique prédominant, avec des personnalités marquantes telles que Diderot ou Rousseau, dont les idées ont servi de base à des novateurs tel que Pestalozzi, des initiateurs de l'école active dans laquelle le sujet et sa propre perception sensorielle sont fondamentaux pour la connaissance de la réalité : «Plus tu utilises de sens dans la recherche de l'être ou des propriétés d'une chose, plus ta connaissance de celle-ci sera exacte » (Pestalozzi, 1932, p. 117).

\section{2 Le constructivisme dans l'enseignement et la recherche}

52 Bien que la pratique et la recherche en éducation peuvent fournir des résultats intéressants quel que soit le point de vue philosophique ou épistémologique, l'essor du constructivisme radical dans l'éducation, malgré les affirmations génériques antidogmatiques, a favorisé un type de pratique et de recherche se réduisant bien souvent à une déclaration de foi constructiviste et à une diatribe contre les méthodes traditionnelles d'enseignement et de recherche. Par ailleurs, en opposition avec la remarque précédente, compte tenu de l'ambiguïté des principes épistémologiques généraux du constructivisme radical et de ses développements - nous pouvons dire que le constructivisme trivial manque de principes épistémologiques généraux - toute pratique éducative et de recherche, de la plus transmissive aux méthodes les plus empiriquement réalistes et expérimentales, peuvent trouver un soutien au moins partiel dans les formulations constructivistes radicales ${ }^{10}$. 
53 L'incohérence du constructivisme épistémologique radical se répète dans le constructivisme pédagogique, transposant aussi dans l'enseignement la confusion et les "fréquentes rechutes dans l'objectivisme de la science traditionnelle, théoriquement rejeté, et dans le dogmatisme » (Martinez Delgado, 1998, p. 184).

Les constructivismes épistémologique et éducatifs se présentent avant tout comme des positions de rejet, au moins déclaratif, vis-à-vis des formes et des méthodes considérées comme réalistes. En particulier, le constructivisme éducatif, plus ou moins radical ou radicalisé, a mis l'accent sur les postulats et orientations méthodologiques suivants :

1. Séparation marquée entre enseignement et instruction (ou entraînement). Selon von Glasersfeld (1991, p. 32) «Il y aura une séparation radicale entre les procédures éducatives que visent à générer compréhension (enseignement, teaching) et celles qui ne sont simplement dirigées qu'à la répétition de comportements (instruction, training) ».

La distinction entre ces deux types d'apprentissage est un lieu commun, pris en compte par les enseignants en général dans le monde de l'éducation. Cependant, leur séparation radicale est une idée qui semble même contraire à l'unité proclamée du sujet dans les principes constructivistes radicaux.

Dans la pratique éducative, cette stricte séparation peut même avoir des effets contreproductifs, car la compréhension de certains concepts progresse souvent avec l'application répétée, utilisant et faisant apparaitre de nouveaux niveaux de compréhension. Certaines idées et certains concepts sont compris pleinement uniquement à travers l'apprentissage de mécanismes d'action répétitifs, grâce aux possibilités de maturation qu'offre un tel entraînement.

58 La séparation stricte entre enseignement et instruction n'est qu'un aspect de la vision dogmatique constructiviste du sujet, exclusivement comme sujet cognitif (rationnel), marginalisant des aspects fondamentaux tels que les aspects émotionnels et affectifs (Carretero, 1993, p. 122).

59 2. Rejet de l'enseignement transmissif. Von Glasersfeld (1991, p. 32) fournit une nouvelle séparation radicale entre deux fonctions du langage :

60 Il est surprenant que le langage ne puisse être utilisé pour transférer n'importe quel type de connaissance et, cependant, qu'il puisse transmettre des instructions constructives à suivre, des instructions supposées adéquates aux caractéristiques personnelles - ou propres au groupe - de l'élève, une réalité, évidemment extérieure au professeur, qui serait cependant connue de lui de façon objective et approfondie.

61 Malgré la transition du je individuel au nous, préconisée entre autres par von Glasersfeld et Foerster, qui en principe ouvrirait la porte de l'intercommunication entre les sujets distincts et de la transmission d'information et d'éléments cognitifs, une grande partie du constructivisme pédagogique est opposée à l'apprentissage par transmission, une opposition qui est renforcée par la fallacieuse équivalence entre l'enseignement par transmission et l'apprentissage par cœur (sans que cela implique aucun mépris de notre part pour le travail de la mémoire).

62 L'attaque contre la transmissibilité de la connaissance suppose, d'une part, un dogmatisme de principe individualiste, et d'autre part, une méfiance envers la validité des principes constructivistes, réduite exclusivement à l'emploi de méthodes ou de rituels considérés comme authentiquement constructivistes. Le fait que la connaissance soit, dans une certaine mesure, construite par le sujet, devrait également s'appliquer à 
l'enseignement par transmission. En ce sens, Millar (1989, p. 589) souligne le problème du constructivisme "pour expliquer comment la plupart des gens dont on pourrait dire qu'ils ont une compréhension de la science ont réussi à l'acquérir » sans avoir suivi « des programmes d'apprentissage structurés selon des lignes constructivistes ».

Le refus catégorique d'accepter la transmission de la connaissance conduirait à nier le caractère social et cumulatif de la connaissance ce qui pourtant nous semble un fait évident.

3. Rejet de l'enseignement et de la recherche empirique et expérimentale (par découverte), de l'induction ${ }^{11}$ et des méthodes d'analyse quantitative. Soutien à l'enseignement et à la recherche par invention déductive et utilisation de méthodes qualitatives.

Il est étonnant que le constructivisme radical rejette l'utilisation de données empiriques et expérimentales car elles peuvent être intégrées sans grandes difficultés à l'expérience personnelle de l'individu ${ }^{12}$, sans aucune référence à une réalité en dehors de celui-ci.

En fait, le positivisme traditionnel et l'empirisme (revendiqué par von Glasersfeld comme un précurseur du constructivisme radical) envisagent l'enseignement et la recherche dans cette direction. L'empirisme constructiviste radical, cependant, place au second plan le flux expérimental personnel, dans la version la plus nettement solipsiste, et fait du noyau du sujet - responsable des idées et des structures qui génèrent la propre expérience du sujet idéal, difficilement différenciable de l'âme des mythologies -, l'unique origine de la connaissance.

67 Pour des raisons similaires, le constructivisme radical pédagogique rejette les méthodes inductives d'apprentissage et de recherche, un rejet qui s'étend aux méthodes quantitatives d'analyse et d'expérimentation scientifique. En revanche, il milite pour l'utilisation de méthodes déductives de connaissance et pour l'analyse qualitative. Les limitations de la déduction, si chère aux théologiens scolastiques, semblent évidentes lorsqu'on essaie d'acquérir des connaissances scientifiques. Piaget (1979, p. 97-98), insiste sur l'importance de l'expérimentation dans l'enseignement actif et dans l'invention même des connaissances :

Les théories scientifiques, la formulation d'hypothèses et les chaînes déductives ne peuvent pas être dissociées de l'observation et de l'étude de la réalité extérieure. Diderot (1981, p. 33) soutient clairement ce point de vue réaliste et matérialiste :

La reconnaissance de cette réalité, constituée d'un grand ensemble de faits indépendants de notre volonté, de nos désirs, imaginations et élaborations théoriques, et dans laquelle est immergée notre propre subjectivité, est un appui fondamental pour la connaissance scientifique. Les manifestations de la réalité permettent non seulement de filtrer nos idées et théories, avec la réfutation ou la falsification poppériennes, mais permet aussi l'émergence inductive d'idées et hypothèses.

70 La confrontation entre nos idées et la réalité extérieure se produit d'une façon particulièrement intense dans l'expérimentation scientifique. Le laboratoire est l'institution scientifique qui permet l'organisation systématique de l'expérimentation et de la recherche en Physique et dans d'autres sciences de la nature et de l'homme. Les modèles et les simulations numériques sont de puissants instruments d'expérimentation virtuelle mais ne doivent pas se substituer au laboratoire dans l'apprentissage et dans la recherche en Physique ou dans d'autres sciences.

71 La conformité de nos idées et connaissances avec la réalité, et surtout avec un certain nombre de perceptions de cette réalité, ne peut pas cependant être tenue pour absolue, 
définitivement acquise et immuable. Malgré le fait que l'espèce humaine et ses individus sont une partie de la nature, les systèmes de perception et de réflexion humains ne couvrent pas la totalité des phénomènes physiques. Une théorie scientifique n'a donc pas, d'un point de vue réaliste et matérialiste, un caractère absolu.

La tendance à faire de nos observations et convictions sur la réalité un absolu est un danger dans le développement des sciences qu'on peut éviter par une attitude générale relativiste et, surtout, en élargissant nos moyens d'observation et d'expérimentation. Il est vrai que le positivisme - dont les principes sont similaires avec ceux du constructivisme radical - a conduit à une certaine parcellisation des faits objets d'étude scientifique et que si on limite l'idée d'observation aux possibilités directes des sens humains, sans chercher de nouveaux moyens d'observation, à la façon d'un positivisme strict et d'un inductivisme tout court, les idées et théories scientifiques prévalentes à une époque peuvent devenir des obstacles (épistémologiques) pour le progrès des sciences. L'expérimentation et le travail de laboratoire demeurent cependant des éléments fondamentaux de l'apprentissage et de la recherche.

4. Apprentissage à partir des idées-connaissances préalables des élèves dans la résolution de problèmes. Alors que dans le constructivisme épistémologique on parle sans tabous de connaissance, dans le constructivisme pédagogique, se montrant dans ce cas plus radical que le constructivisme épistémologique, on évite ce terme et on le remplace par idées, sans doute considérant que le terme de connaissance a des connotations réalistes, lesquelles sont à éviter pour une théorisation constructiviste.

La notion d'idées préalables, comme tant d'autres utilisées comme slogans, d'une part, est plus complexe que ne le suggère sa simple proclamation, et d'autre part, n'est pas l'apanage exclusif des pédagogues constructivistes. Les idées préalables sont seulement reconnaissables d'une manière approximative, parfois de façon grossière. Comment donc le constructivisme, qui nie qu'on puisse obtenir une connaissance d'une réalité objective, peut-il prétendre avoir accès à une connaissance pleine et entière des idées d'autres personnes, à savoir les élèves ou les étudiants? Une telle prétention ne constitue-t-elle pas une forme extrême de réalisme présupposé par le constructivisme radical et que la plupart des réalistes, par prudence, se garderaient d'adopter? En outre, débuter toute séance d'enseignement par l'établissement d'une carte des idées préalables des élèves, avant de faire une activité ou de communiquer n'importe quelle connaissance déterminée, peut présenter un intérêt du point de vue de la psychologie de l'apprentissage, mais n'a pas un intérêt direct du point de vue de l'activité quotidienne.

Le programme constructiviste consistant à déterminer les idées des étudiants et à les engager dans la résolution de problèmes à résoudre, peut être considéré non seulement comme l'expression d'une position extrêmement réaliste, mais aussi comme un retour, au moins partiel, au behaviourisme.

76 Faire de la résolution de problèmes une caractéristique distinctive exclusive au constructivisme est une appropriation inacceptable, comme c'est le cas aussi de l'appropriation par le constructivisme de la pédagogie active, traitée précédemment.

5. Impulsion de plans pour l'éducation et de réformes des systèmes d'enseignement basés sur ou inspirés par le constructivisme, favorisant ainsi une hégémonie constructiviste appuyée en partie par les administrations scolaires et académiques. L'attitude sectaire et élitiste des publicistes et des autorités académiques et éducatives envers les habitudes dites conservatrices d'une grande partie du corps enseignant a été un facteur 
supplémentaire de la détérioration de l'enseignement et de la situation des enseignants, détérioration qui a des racines profondes dans l'évolution économico-sociale et l'idéologie qui a conduit à la grande crise du début du XXIe siècle ${ }^{13}$.

78 Paradoxalement, l'échec des systèmes éducatifs, en partie lié à la mise en oeuvre de lignes éducatives constructivistes plus ou moins radicales, est utilisé dans certains secteurs influents pour justifier de faire appel au constructivisme radical. Par là même, on semble choisir une sorte d'homéopathie sans aucun contrôle de rationalité, qui peut être particulièrement destructrice pour la résolution de l'échec scolaire, parce que les méthodes constructivistes semblent ne pas être les plus propices au développement cognitif des élèves les moins motivés pour les études.

79 L'enthousiasme du réformisme constructiviste a abouti à des slogans du genre de celui lancé par Jan de Lange, directeur de l'Institut Freudenthal (van Reeuwijk, 1992, p. 516) : "La meilleure façon d'enseigner est de ne pas enseigner ».

80 Ce genre de proclamations, même si elles sont en partie fondées, a joué un rôle de fonction destructrice sur les enseignants non universitaires, comme l'on suggéré les " constructivistes radicaux» Arcavi et Shoenfeld (1992, pp. 333-334) se référant aux difficultés théoriques et pratiques du constructivisme :

81 Le radicalisme constructiviste, en niant que la connaissance puisse être transmise, comporte une attitude éducative anti-didactique. Si la connaissance est une construction purement individuelle, il n'y a pas de sens à vouloir enseigner des contenus concrets, du moins si l'on s'en tient strictement aux principes du constructivisme radical.

82 La prise de distance du constructivisme vis-à-vis de la didactique se retrouve aussi chez Socrate, qui dans le Ménon défend « un modèle pédagogique non-didactique » (Nola, 1998, p. 34), ce qui conduit Nola à conclure que «Socrate et Platon sont les premiers constructivistes en éducation » (ibid., p. 35).

83 Mais, comme on l'a déjà indiqué, le manque de cohérence entre la théorie et la pratique est l'une des caractéristiques remarquables tant de la position de Socrate-Platon que du constructivisme radical. Nous avons déjà souligné le fait que Socrate, par le moyen d'une construction géométrique adéquate, trouve un moyen didactique pour enseigner à l'esclave de Ménon la solution du problème posé. Von Glasersfeld lui-même analyse du point de vue didactique la meilleure façon d'enseigner le théorème de Pythagore aux élèves du secondaire, en utilisant aussi une illustration graphique ( «le modèle perceptuel ») comme un appui à la démonstration du théorème (von Glasersfeld, 2009, p. 229). Cette contradiction entre les aspects doctrinaux et l'activité pratique se retrouve aussi souvent dans des recherches dites constructivistes sur l'apprentissage, en particulier en physique. C'est l'une des raisons pour ne pas mépriser des travaux simplement parce qu'ils s'affichent comme étant constructivistes.

Le soutien officiel pour l'enseignement constructiviste semble s'accompagner d'un mépris de la rigueur des connaissances, en donnant la priorité à un conformisme social intégrateur, basé en partie sur des rituels et des réalités virtuelles publicitaires. La contrestimulation de l'élan vers la profondeur de la connaissance, la dégradation de la soif de savoir, se reflètent même dans les sauts de principes éducatifs à d'autres principes officiels, sans justification ni débat théorique, dans la transformation des théories en simples béquilles sans contenu réel. Un exemple récent de cette déconsidération envers l'idée de vérité et envers la cohérence du discours, a été la subite relégation des mêmes slogans constructivistes par l'approche en termes de compétences éducatives, dont 
l'orientation pragmatique n'est guère justifiée sur le plan théorique, une subite prescription des administrations éducatives centrales européennes, acceptées avec soumission et exaltées par des autorités et des idéologues éducatifs de différents pays.

\section{Le constructivisme et le postmodernisme : fonctions idéologiques}

Le constructivisme radical fait partie des tendances postmodernes qui portent leur critique des grands discours jusqu'à la négation de toute connaissance objective, en particulier des faits sociaux ${ }^{14}$. Le fait que la métaphysique constructiviste du sujetprolongement de la pensée moderniste idéaliste, la pensée moderne prédominante puisse être vue comme un des grands discours critiqués par une partie des courants postmodernes, n'est pas un obstacle à l'intégration du constructivisme radical au sein des orientations postmodernes, peu exigeantes du reste en matière de cohérence.

Les principaux courants postmodernes nient l'existence d'une réalité objective extérieure à l'individu et la substitue par une sorte de réalité intermédiaire entre le sujet et une réalité indépendante ou rendue indépendante : le texte narratif. Cette réalité en grande partie matérialisée, présente certaines caractéristiques d'objectivité mais introduit, en même temps, la virtualité de la construction narrative, présence virtuelle qui tend à devenir hégémonique à travers la croissante informatisation de l'information, de la communication, de l'enseignement et d'une grande partie des activités de travail.

Malgré la phraséologie glorificatrice de cette nouvelle réalité comme instrument d'émancipation, avec pour le moins des marques factuelles apparentes telles que les mouvements populaires dans certains pays arabes, il y a des indices suffisants pour croire que ces réseaux et la communication électronique se trouvent principalement sous le contrôle des grands pouvoirs impériaux, économiques et politiques. Les différents types de sites qui se succèdent, annonçant l'obtention d'une participation pleinement démocratique et égalitaire de tous les participants et l'apparition d'un type d'enseignement totalement nouveau, basé sur le travail en réseau, sont effectivement de puissants instruments de communication, d'information et de travail, mais sont souvent employés comme voile pour cacher le cours réel des événements et servent à la manipulation, l'isolement réel et l'oppression idéologique et émotionnelle des individus. Bien que les potentialités économiques des ordinateurs et des réseaux soient extraordinaires pour la vie des personnes et qu'il serait insensé d'essayer de se passer de ces outils TIC, ils ne parviennent pas cependant à remplacer complètement les réseaux traditionnels de communication personnelle et de la relation avec le monde extérieur objectif, et dans les cas où ils réussissent à déplacer ces réalités, leurs effets s'avèrent dévastateurs.

Ces instruments électroniques ne parviennent pas non plus à masquer totalement le fait que, derrière un discours apparemment technologique, neutre, même bénéfique pour la libération individuelle, demeure un combat féroce pour le contrôle des sources d'énergie, des matières premières et pour le contrôle des marchés. L'analyse des plus récentes formes de domination, le soutien technologique de ces dernières et leur imbrication dans une mentalité qui vise à remplacer des réalités d'une base objective par des réalités virtuelles, créées par certains organismes sociaux, nous permettent d'interpréter la signification sociale, politique et idéologique de théories comme le constructivisme 
radical et d'autres dérivés, dont l'ample et rapide propagation semble difficilement compatible avec le principe exclusif de la construction individuelle.

Le constructivisme, tout comme d'autres courants postmodernes, prétend être une théorie flexible, tolérante et libératrice ${ }^{15}$, affirmation qui se justifierait d'un côté par sa revendication de l'individu isolé (ce qui permet d'utiliser le discours du néolibéralisme dominant) et, d'autre part, par le scepticisme et le relativisme qu'il présuppose. Le constructivisme radical disqualifie le réalisme comme une position conduisant au fanatisme et à la tyrannie. Maturana (1991, p. 49), après avoir caricaturé la position des scientifiques réalistes avant l'expansion de la nouvelle foi constructiviste ${ }^{16}$, expose sa vision des « deux grands dangers spirituels » pour une personne (1991, p. 51) :

Le réalisme, au moins dans sa conception matérialiste, n'affirme pas que notre connaissance est un reflet complet et fidèle d'une réalité extérieure au sujet, fournissant ainsi une vérité absolue, mais soutient que les hypothèses et les théories sont l'objet d'un processus illimité de vérification. Au contraire, le constructivisme radical conduit à un dogmatisme individualiste selon lequel la vérité de chaque personne, aux différentes étapes des constructions personnelles, est, pour reprendre l'expression de la citation précédente, «aveugle à ses circonstances ». Ces circonstances dépendent pourtant de la réalité extérieure au sujet, laquelle réalité est occultée par le constructivisme radical ${ }^{17}$. La référence de Maturana au "spirituel» exprime d'ailleurs l'arrière-plan idéaliste et religieux du constructivisme radical ${ }^{18}$.

Le deuxième problème indiqué par Maturana dans la citation ci-dessus a des conséquences idéologiques, sociales et politiques évidentes. L'idée de la responsabilité exclusive de l'individu sur son propre devenir et sur son futur est fréquemment soutenue par les auteurs constructivistes radicaux. Von Glasersfeld (1995, p. 19) semble attacher plus d'importance à cet aspect socio-politique qu'à l'aspect purement épistémologique, lorsqu'il décrit le constructivisme radical comme une orientation qui :

Un autre élément, d'ordre économique, rejoint les approches théoriques constructivistes et postmodernes: l'hégémonie du capitalisme financier dans le capitalisme mondial. Le capitalisme financier suppose un pas de plus par rapport à ce que Marx a appelé le fétichisme de l'argent et intensifie le sens virtuel représenté par l'argent, au point d'apparaître et d'agir comme la forme unique ou principale de mise en valeur économique, indépendante du travail. Le détachement du bénéfice économique de la production matérielle et du travail transforme le système du capital financier en une forme d'économie virtuelle, en accord avec des tendances similaires du constructivisme et du postmodernisme. Cependant, ce système économique, en particulier sa composante financière, se heurte à des difficultés qui s'avèrent d'une extrême gravité, voire autodestrustructrices. C'est ce que l'on observe actuellement avec la crise économique qui est en grande partie le résultat de l'hégémonie de constructions fictives et de bulles financières. L'économie financière, en dépit de sa position dominante actuelle dans l'ensemble du système économique, ne peut pas se passer des bénéfices matériels palpables qui sont encore générés à partir de l'exploitation de la main d'œuvre, des avantages organisationnels, technologiques et de l'influence politique d'entreprises impliquées dans les processus de mondialisation.

93 Le triomphe du virtuel sur le réel en économie a son pendant cognitif, dans un déplacement similaire, opéré par le constructivisme radical, de la réalité matérielle, extérieure à l'individu, vers les créations virtuelles individuelles, considérées comme la seule source de la connaissance. Ces similarités et cette analogie entre les tendances 
économiques et certaines lignes théoriques permettent de formuler l'hypothèse de l'existence d'une liaison entre les deux sphères, économique et cognitive, qui peut être formulée en termes de classes sociales.

Jameson, critique culturel qui a tenté d'adapter le marxisme aux courants postmodernes et à un constructivisme radical dialectique, a attribué au réalisme épistémologique un caractère de classe bourgeois (Jameson, 2009, p. 322). Il a également soutenu l'idée du lien entre le postmodernisme et le capitalisme tardif, quoique cette dernière dépendance de classe semble moins stricte pour Jameson que pour le cas du réalisme et la classe bourgeoise. Si nous accordons une certaine validité à l'analyse en termes de classes sociales dans notre société, il semble recevable de caractériser le réalisme comme une idéologie de la bourgeoisie, ainsi que le soutient Jameson, mais en même temps, il semble également justifié de remarquer les tendances et alignements de classe du constructivisme radical, dans le cadre des courants postmodernes.

Prenant en compte la confluence remarquée entre les théorisations épistémologicoéducatives (avec le transfert de la responsabilité exclusivement vers l'individu, évitant ainsi toute considération des aspects négatifs du système socio-économique et des privilèges de groupe) et les tendances socio-économiques prépondérantes, nous pouvons caractériser le constructivisme radical comme une idéologie de la classe financière capitaliste. La prédominance de ce secteur de la classe capitaliste, favorisée par des approches théoriques qui justifient leurs formes de domination, peut être l'une des causes du succès précipité de la pensée constructiviste radicale. Cela ne signifie pas que le constructivisme radical soit la seule idéologie de la classe capitaliste financière, notamment de ses secteurs oligarchiques, mais que dans les circonstances présentes, le constructivisme radical sert d'appui au maintien des intérêts de cette classe, qui analysée de l'intérieur comprend le secteur supérieur des cadres financiers, ne faisant pas partie à l'origine de la classe capitaliste à proprement parler.

Les considérations précédentes n'impliquent pas que, d'une façon simpliste et manichéenne, on puisse attribuer directement à une philosophie ou une épistémologie réalistes une signification émancipatrice des classes opprimées. En fait un réalisme empiriste tout court fait partie d'une solide attitude conservatrice (les choses sont - et seront - telles qu'elles sont maintenant). La fonction de classe des positions philosophicoidéologiques, surtout dans un sens libérateur, est un des problèmes fondamentaux à étudier et débattre.

97 Popper (1982, p. 99) porte un jugement sévère sur la pensée platonicienne qui pourrait aussi être appliquée à quelques positions constructivistes et postmodernes :

\section{Conclusions}

1. Malgré le mépris du constructivisme radical pour la théorie socratique de la connaissance, nous pouvons considérer que la maïeutique socratique est une forme de constructivisme, aux principes aussi radicaux que ceux annoncés par von Glasersfeld. La proximité entre le constructivisme radical et la théorie socratique, exposée notamment dans le Ménon, révèle les principes épistémologiques mais aussi les incohérences et les confusions de chacune de ces positions.

2. Les incohérences du constructivisme épistémologique radical se retrouvent dans le constructivisme éducatif radical, lequel ajoute de nouvelles incohérences. L'annexion au 
constructivisme d'une grande part de ce qu'a été la pédagogie active est injustifiée d'un point de vue théorique et abusive d'un point de vue pratique. La non prise en compte de données empiriques et de l'expérimentation scientifique dans l'apprentissage et la recherche est un obstacle propre au constructivisme pédagogique et cognitif en général, qui promeut un style superficiel et arbitraire de recherche, où le récit subjectif se substitue à quelques conditions minimales de contraste et de contrôle sur la théorie.

3. Assez souvent, le constructivisme radical s'approprie comme une partie de la théorie même, des idées appartenant à d'autres auteurs dans une vision biaisée de ces mêmes idées, (c'est particulièrement le cas de Piaget). Dans d'autres cas (Socrate), la version constructiviste tend à la mise à distance, parfois forcée, des idées des auteurs. Bien que selon les principes constructivistes, la connaissance, et par conséquent aussi, les idées d'autres auteurs, soit personnelle et donc subjective, les idéologues du constructivisme se montrent plus strictement objectivistes par rapport aux critiques qui leur sont adressées et qu'ils considèrent souvent comme le produit d'une erreur de compréhension.

4. Idéologiquement le constructivisme fait partie des courants postmodernes, contraires à l'établissement de critères d'objectivité, en particulier dans la sphère sociale et dans les grands discours de la pensée moderne. La prise de distance du constructivisme radical vis-à-vis des grands discours de la modernité est cependant partiel, puisque l'accent sur le sujet comme unique réalité essentielle est précisément un prolongement de la pensée idéaliste moderne, précisément le créateur des discours de la modernité aux prétentions les plus absolues.

102 L'objectif du constructivisme radical est de placer la responsabilité de tous les événements vécus par l'individu dans le sujet lui-même. Il a clairement des connotations idéologiques. En termes généraux, ce principe peut être intégré à la pensée néolibérale. Plus précisément, on peut considérer que le constructivisme radical est une idéologie de la classe financière aujourd'hui dominante, en particulier de son secteur oligarchique, dans un contexte de mondialisation de sa domination, de fragmentation et d'individualisation des secteurs sociaux dominés.

103 5. La critique du constructivisme radical et de sa pression hégémonique ne doit pas empêcher la valorisation positive de recherches et d'applications pédagogiques rattachées à des principes constructivistes radicaux ${ }^{19}$. Dans tous les cas, le respect de la liberté de recherche, sans obédiences inconditionnelles, doit prévaloir ainsi que celui de la liberté des choix méthodologiques pédagogiques, dans le respect des élèves et des étudiants. L'intérêt d'une recherche ou d'une méthode d'enseignement ne réside pas fondamentalement dans le paradigme invoqué. Notre désaccord avec l'idéalisme socratique-platonicien, par exemple, ne nous empêche pas d'apprécier l'emploi du dialogue comme un puissant instrument d'apprentissage.

\section{BIBLIOGRAPHIE}

Arcavi, A. et Shoenfeld, A. H., Mathematics Tutoring Through a Constructivist Lens ; The Challenges of Sense-Making, Journal of Mathematical Behavior, 11 (4), 1992, p. 321-335.

Baillargeon, N., Misère du constructivisme, À bâbord. Revue sociale et politique, 9 (avril-mai), 2005. Bkouche, R., Quelques remarques à propos de l'enseignement de la géométrie, Repères IREM (IREM de Lille), 26, 1997, p. 49-71. 
Canto-Sperber, M., Introduction. Dans Platon, Ménon. Paris : Flammarion. 1993.

Carretero, M., Constructivismo y educación. Zaragoza : Edelvives. 1993.

Comenius, J. A., The Way of Light. Liverpool: Liverpool The University Press. 1938.

Diderot, D., Pensées sur l'interprétation de la nature (đEuvres Complètes, t. IX). Paris : Hermann. 1981.

Ernest, P., Varieties of Constructivism: Their Métaphores, Epistemologies and Pedagogical Implication. Dans Preece, P. and Fox, R. (eds.), Perspectives on Constructivism. Media and Ressources Centre, University of Exeter School of Education. 1996, p. 73-92.

Fox, R., Piaget on learning. Preece, P. and Fox, R. (eds.), Perspectives on Constructivism. Media and Ressources Centre, University of Exeter School of Education. 1996, p. 23-37.

Glasersfeld, E., von, An Introduction to Radical Constructivism. Dans Watzlawick, P. (ed.), The Invented Reality. New York : Norton. 1984, p.17-40.

Glasersfeld, E., von, Constructivism in Education. Dans A. Lewy (ed.), The International Encyclopedia of Curriculum. Oxford : Pergamon Press. 1991, p. 31-32.

Glasersfeld, E., von. Radical Constructivism : A Way of Knowing and Learning. London and Washington : The Falmer Press. 1995.

Glasersfeld, E., von, Introduction: Aspects of Constructivism. Dans Fosnot, C. T. (ed.), Constructivism : Theory, Perspectives, and Practice. New York and London : Teacher College, Columbia University. 1996, p. 3-7.

Hardy, M. D. et Taylor, P. C., Von Glasersfeld's Radical Constructivism : A Critical Review, Science and Education, 6 (1-2), 1997, p. 135-150.

Hatwell, Y., À propos des notions d'assimilation et d'accomodation dans les processus cognitifs. Dans J. B. Grize et al. (eds.), Psychologie et épistémologie génétique : thèmes piagetiens. Paris : Dunod. 1966, p. 127-136.

Jameson, F., Valences of the Dialectic. London: Verso. 2009.

Kelly, G., The Psychology of Personal Constructs. Vol. 1 A Theory of Personality. New York : Norton and Company. 1955.

Kelly, G., Clinical Psychology and Personality. The Selected Papers of George Kelly. New York : John Wiley and Sons. 1969.

Kelly, G. J., Research Traditions in Comparative Context : A Philosophical Challenge to Radical Constructivism, Science Education, 81(3), 1997, p. 355-375.

Kilpatrick, J., Lo que el constructivismo puede ser para la educación matemática, Educar, 17, 1990, p. 37-52.

Krippendorff, K., Reconstructing (Some) Communication Research Methods. Dans F. Steier, (ed.), Research and Reflexivity. London: Sage Publications. 1991, p. 115-142.

La Capra, D., Writing history, writing trauma. Baltimore : The Johns Hopkins University Press. 2001. Lerman, S., Intersubjectivity in Mathematics Learning: A Challenge to the Radical Constructivist Paradigm ? Journal for Research in Mathematics Education, 27 (2), 1996, p. 133-150.

Martínez Delgado, A., No todos somos constructivistas, Revista de Educación, 315, 1998, p. 179-198.

Martínez Delgado, A., Radical Constructivism: Between Realism and Solipsism, Science Education, 86 (6), 2002, p. 840-855. 
Matthews, M. R., Challenging NZ Science Education. Palmerston North: The Dunmore Press. 1995. Maturana, H, R., Science and Daily Life : The Ontology of Scientific Explanations. Dans F. Steier (ed.) Research and Reflexivity. London: Sage Publications. 1991, p. 30-52

Millar, R., Constructive criticisms, International Journal of Science Education, 11 (special issue), 1989, p. 587-596.

Nola, R., Constructivism in Science and Science Education : A Philosophical Critique, Science and Education, 6 (1-2), 1997, p. 55-83.

Nola, R., Constructivism in Science and Science Education : A Philosophical Critique. Dans M. R. Matthews (ed.) Constructivism in Science Education: A Philosophical Examination. Dordrecht: Kluwer Academic Publishers. 1998, p. 31-59.

Ortega y Gasset, J., Obras Completas, Vol. 1 (1902-1915). Madrid : Taurus, Fundación José Ortega y Gasset. 2004

Osborne, J. F., Beyond Constructivism, Science Education, 80 (1), 1996, p. 53-82.

Perrenoud, Ph., Pour ou contre la gravitation universelle? Le constructivisme n'est ni un dogme, ni une mode. Disponible sur internet : <http://www.unige.ch/fapse/SSE/teachers/perrenoud/php_main/php_2003/2003_22.html>. 2003.

Pestalozzi, E., Como enseña Gertrudis a sus hijos. Madrid: Espasa-Calpe. 1932.

Piaget, J., Adaptación vital y psicología de la inteligencia. Madrid : Siglo veintiuno. 1974/1978.

Piaget, J., A donde va la educación. Barcelona : Teide. 1979.

Piaget, J., La toma de conciencia. Madrid: Morata. 1974/1985.

Plato, The Trial and Death of Socrates. Four Dialogues. New York : Dover Publications. 1992.

Platon, Ménon. Paris : Flammarion. 1993.

Popper, K. R., La Sociedad abierta y sus enemigos. Barcelona/Buenos Aires : Paidós. 1982.

Reeuwijk, M. van, The Standards Applied: Teaching Data Visualization, Mathematics Teacher, 85 (7), 1992, p. 513-518.

Russell, B., History of Western Philosophy. London : Unwin Paperbacks. 1979.

Schoenfeld, A. H., Radical Constructivism and the Pragmatics of Instruction, Journal for Research in Mathematics Education, 23 (3), 1992, p. 290-295.

Segal, L., The Dream of Reality. Heinz von Foerster's Constructivism. New York and London: W. W. Norton. 1986. 


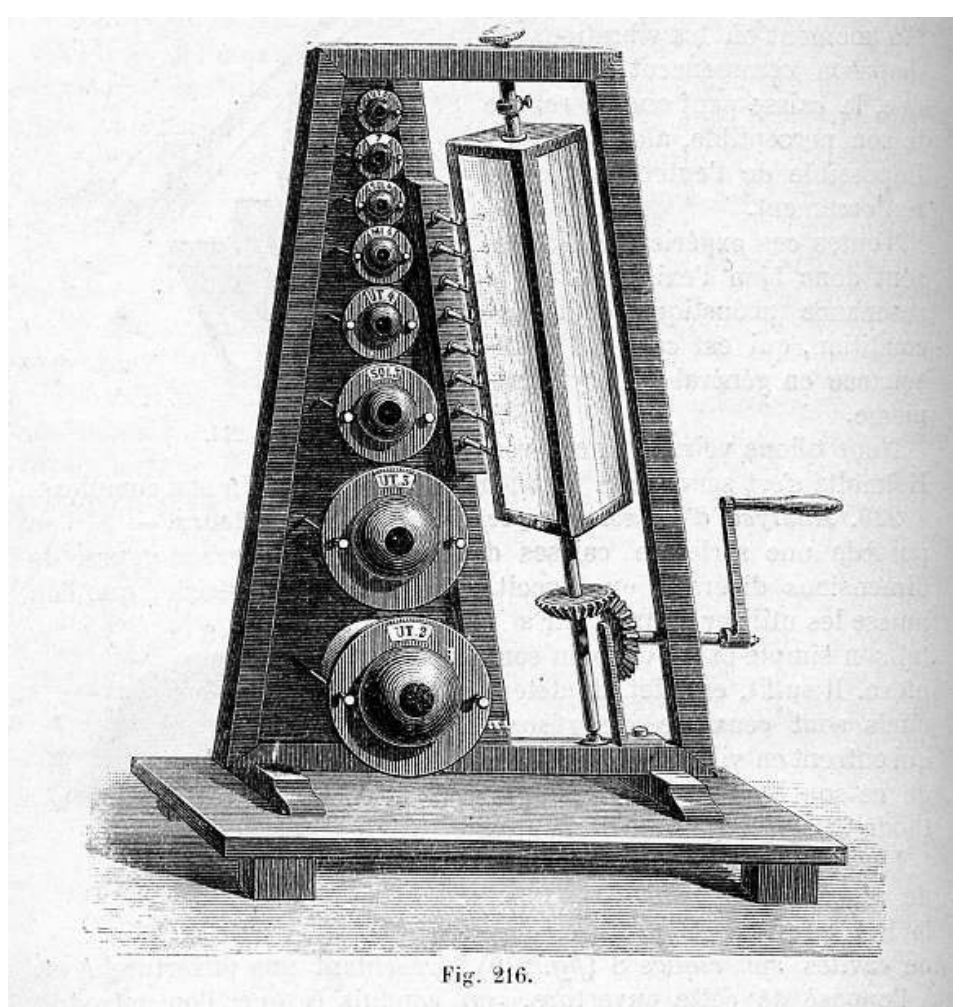

Analyse d'un son complexe par des résonateurs : expérience avec des capsules manométriques dont on observe les flammes dans un miroir tournant.

Nouveau cours de physique élémentaire, troisième partie, classes de mathématiques A et B. Faivre-

Dupaigre, J., Carimey, E., deuxième édition, Masson, 1909. Source CEDRHE, 22932, p. 288.

\section{NOTES}

1. Cet article initialement écrit par l'auteur en espagnol a été traduit en français par Isabelle Aliaga (IUFM de Montpellier, UM2), avec le soutien financier du pôle recherche de l'IUFM de Montpellier.

2. De nombreux auteurs accordent un rôle fondamental à von Glasersfeld dans la création et le développement du constructivisme, en particulier du constructivisme radical : «le principal auteur représentatif du "constructivisme radical" [...]» (Hardy and Taylor, 1997, p. 135); «la position constructiviste radicale est représentée le mieux par von Glasersfeld » (Kelly, G. J., 1997, p. 356); « le plus important représentant du constructivisme aux Etats-Unis [...] [qui] offre la plus élaborée et cohérente base pour une analyse initiale [du constructivisme]» (Kilpatrick, 1990, p. $39)$; « [...] dans sa forme moderne le constructivisme radical a été élaboré de la façon la plus complète en termes épistémologiques par von Glasersfeld » (Ernest, 1996, p. 79); « [...] Glasersfeld est l'un des principaux représentants d'une version radicale du constructivisme comme théorie de la connaissance scientifique aussi bien que comme un guide pour l'éducation scientifique » (Nola, 1997, p. 69). L'absence d'une réalité extérieure qui puisse être connue dans une certaine mesure par le sujet, et donc faire de celui-ci l'unique auteur de la connaissance, indépendamment de toute réalité extérieure à l'individu lui-même peut être considérée comme le principal postulat de constructivisme radical (von Glasersfeld, 1984, p. 24).

3. Une défense exemplaire du constructivisme trivial, en référence à la gravitation universelle, est le texte suivant de Perrenoud (2003) : « [...] la gravitation universelle est une loi de la nature, dont on ne peut que prendre acte. [...] Le constructivisme n'est ni une mode, ni une doctrine. Ce n'est pas non plus, en soi, une démarche pédagogique. C'est une "loi" de l'apprentissage humain, 
qui dit que tout apprentissage passe par une activité mentale du sujet, une activité de réorganisation du système de schèmes et de connaissances existant ».

4. Que le sujet construise sa propre connaissance, jusqu'à un certain point, peut être admis sans beaucoup de souci. Le problème est de savoir s'il la construit seul, s'il s'agit seulement d'une invention du sujet ou si elle est aussi le produit d'une réalité extérieure. Ce constructivisme partiel ou trivial, loin de constituer un paradigme, peut être vu comme une banalité que les seuls partisans de la tabula rasa nieraient.

5. Socrate décrit dans le Phédon (Platon, 1992, p. 96) le glissement vers le réalisme d'Anaxagore, un glissement que l'on peut remarquer dans l'évolution des théories empiristes et aussi dans le développement théorique $\mathrm{du}$ constructivisme radical: «[...] Anaxagore [...] l'esprit est l'organisateur et la cause de toutes choses [...] S'il en est ainsi, me dis-je, l'esprit ordonnateur dispose tout et place chaque objet de la façon la meilleure [...] Je pensais qu'il me dirait d'abord si la terre est plate ou ronde et après cela qu'il m'expliquerait la cause et la nécessité de cette forme, en partant du principe du mieux, et en prouvant que le mieux pour elle, c'est d'avoir cette forme, et s'il disait que la terre est au centre du monde, qu'il me ferait voir qu'il était meilleur qu'elle fût au centre [...] Je n'aurais jamais pensé qu'après avoir affirmé que les choses ont été ordonnées par l'esprit, il pût leur attribuer une autre cause que celle-ci [...] Mais je ne tardai pas à tomber du haut de cette merveilleuse espérance. Car, avançant dans ma lecture, je vis un homme qui abandonna l'usage de l'esprit et de tout principe d'ordre et qui mit à sa place l'air, l'éther, l'eau et d'autres choses aussi absurdes ! Il me sembla que c'était exactement comme si l'on disait que Socrate fait par l'esprit tout ce qu'il fait et qu'ensuite, essayant de dire la cause de chacune de mes actions, on soutînt d'abord que, si je suis assis en cet endroit, c'est parce que mon corps est composé d'os et de muscles [...]».

6. Nola (1998, p. 36) affirme à cet égard : «Comme l'épisode avec le garçon l'illustre, le processus d'élimination des erreurs et d'aboutissement à la connaissance par raisonnement présente des éléments constructivistes ».

7. À ce propos, nous avons mené une expérience avec des élèves âgés de 15 ans. Lors d'une première étape, le professeur représentait cet extrait du Ménon avec les élèves, lesquels suivaient de façon spontanée le dialogue avec une fidélité surprenante au texte original. Lors d'une deuxième étape, ils le lisaient directement. Très majoritairement ( $80 \%$ de la classe), les élèves se sont opposés à l'affirmation selon laquelle eux-mêmes, ou l'esclave, auraient trouvé la solution d'eux-mêmes. Il convient aussi de relever l'opinion exprimée de vive voix par quelques élèves de 12 ans, soutenue avec enthousiasme par le reste de la classe, selon laquelle l'esclave, dans le dialogue initié par Socrate, "ne fait que manifester son accord avec ce que Socrate développe ».

8. «Le point de départ de Piaget réside donc dans la continuité qu'il voit entre les processus biologiques d'adaptation de l'organisme au milieu extérieur, et les processus psychologiques de l'intelligence qui assurent, sur un autre plan, la structuration de ce milieu [...]» (Hatwell, 1966, p. 127-128).

9. Piaget a été un artisan majeur, directement ou indirectement de trois grands mouvements de réforme de l'éducation dans la seconde moitié du XXe siècle, avec un fort soutien public : la mise en œuvre, avec certaines rigidités, de la théorie des étapes éducatives en conformité avec les stades du développement en accord avec l'organisation structurelle de la pensée de l'enfant, la réforme des mathématiques modernes associée à l'essor du formalisme en mathématiques, notamment par le groupe Bourbaki ces deux grands mouvements réformistes sont décrits avec plus de détail par Bkouche, 1997, pp. 56-57) - et finalement les réformes constructivistes de la fin du XXe siècle. Fox (1996, p. 23-24) remarque à propos de la théorie des stades de Piaget: «La théorie des stades de Piaget devint peut-être la partie la plus célèbre de toute sa théorie et approximativement entre 1960 et 1980 une énorme quantité de recherches furent effectuées pour la tester [...] Elles n'ont en général pas très bien survécu au déluge de critiques dirigées contre elles ». 
10. La relative indépendance que nous avons fait remarquer entre la théorie générale épistémologique constructiviste radicale et l'approche de l'enseignement et de la recherche a été mise en avant, dans le champ constructiviste même, par Schoenfeld (1992, p. 294).

11. Signalons que le professeur Josef Klauer, dont les travaux sur l'apprentissage par induction ne s'accordent pas avec le modèle constructiviste à la mode, a éprouvé des difficultés à publier sa critique du constructivisme.

12. Krippendorff (1991, p. 141) indique, dans ce sens, la possibilité d'intégrer des formes traditionnelles de recherche au sein du constructivisme: «[...] J'ai essayé de montrer que la méthodologie constructiviste nécessaire pour créer des théories sociales viables et fiables n'a pas besoin de se débarrasser des méthodes traditionnelles d'enquête sociale, mais doit les embrasser dans un discours autoréflexif ».

13. La citation suivante du psychologue cognitiviste Herbert A. Simon est un résumé sévère des aspects négatifs du constructivisme radical en éducation (Baillargeon, 2005) : « S’il n’y a pas eu de progrès constant en éducation, c'est parce qu'en ce domaine on s'est contenté d'aller d'une position simpliste à une autre [...] Actuellement, le constructivisme radical est un exemple d'un tel extrémisme simpliste, et certains de ses promoteurs font preuve d'une attitude antiscientifique tellement marquée que, si celle-ci devait se répandre, il faudrait renoncer à tout espoir de progrès en éducation ».

14. La position ontologique du constructivisme radical s'accorde avec celle de philosophes postmodernes comme Rorty (1989), pour qui «la réalité est indifférente à notre description d'elle " (Osborne, 1996, p. 58).

15. La publicité grandiloquente du constructivisme, aussi bien que l'incohérence de ses principes, sont pointées dans l'affirmation suivante de Segal (1986, p. 4): «En dernière analyse, la préoccupation morale du constructivisme est de réduire les monstres de la raison - le fascisme, le génocide, la guerre nucléaire, et le totalitarisme - en révélant la nature du rêveur ».

16. Maturana (1991, p. 49) : "Nous les scientifiques [les scientifiques traditionnels] sommes concernés par des faits objectifs, nous disons, et de ce fait nos déclarations et explications sont exempts de fanatisme et de préjugés [...] cela n'est que partiellement vrai ».

17. Le fait que ces circonstances soient extérieures au sujet est mis en avant dans le célèbre aphorisme de Ortega y Gasset (2004, p. 757) « je suis moi et ma circonstance ».

18. La Capra (2001, p. 57) considère à cet égard: «Le constructivisme radical pourrait être interprété comme une forme de créationnisme laïque dans lequel l'être humain devient un fondement ultime et le dépôt déplacé de pouvoirs quasi-divins ».

19. L'absence d'une correspondance stricte entre théories générales, d'un côté, et leurs développements théoriques et applications pratiques, de l'autre, conduit même Millar (1989, p. 589) à affirmer que « un modèle constructiviste d'apprentissage n'implique pas cependant un modèle constructiviste d'instruction ". Le progrès des sciences, d'ailleurs, s'est produit dans le cadre de différents paradigmes. Une abondante littérature indique aussi la difficulté, ou même l'impossibilité, de la réfutation d'un paradigme par un autre. Par exemple, selon Lerman (1996, p. 138), la psychologie individualiste et mentaliste ne peut pas être réfutée par la psychologie culturelle et sociale et inversement. 


\section{RÉSUMÉS}

Cet article vise à mettre en évidence l'existence d'une profonde proximité entre le constructivisme radical et l'épistémologie socratique - cette proximité étant niée par d'éminents partisans du constructivisme radical. Il propose une critique de la méthode d'enseignement exposée dans le Ménon de Socrate. Il montre que le constructivisme radical constitue une version biaisée du constructivisme de Kelly et de Piaget. Il discute également les conséquences et formulations pédagogiques du constructivisme, souligne leurs incohérences et propose une critique des aspects idéologiques du constructivisme radical.

This paper aims at pointing out the existence of a deep proximity between radical constructivism and the Socratic epistemology - this proximity being denied by prominent supporters of radical constructivism. It makes a criticism of the teaching method exposed in the Meno of Socrates. It shows that radical constructivism is a biased version of Kelly's and Piaget's constructivism. It deals also with the pedagogical consequences and formulations of constructivism, underlines their inconsistencies, and proposes a criticism of the ideological aspects of radical constructivism.

\section{INDEX}

Mots-clés : constructivisme pédagogique, constructivisme radical, critique du constructivisme, idéologie constructiviste, Ménon, Socrate

Keywords : constructivist ideology, criticism of constructivism, Meno, pedagogical constructivism, radical constructivism, Socrates

\section{AUTEUR}

\section{ALBERTO MARTÍNEZ DELGADO}

IES Tartesos, Camas (Seville), Espagne 